\title{
Orbitofrontal cortex lesions result in abnormal social judgements to emotional faces
}

\author{
Megan L. Willis ${ }^{\mathrm{a}, \mathrm{c}, *}$, Romina Palermo ${ }^{\mathrm{a}, \mathrm{b}}$, Darren Burke ${ }^{\mathrm{c}}, \mathrm{Ky} \mathrm{McGrillen}^{\mathrm{d}}$, Laurie Miller ${ }^{\mathrm{e}, \mathrm{f}}$ \\ a Macquarie Centre for Cognitive Science (MACCS), Macquarie University, Sydney, Australia \\ b Department of Psychology, Australian National University, Canberra, Australia \\ ' School of Psychology, University of Newcastle, Ourimbah, Australia \\ ${ }^{\mathrm{d}}$ Radiology Department, Royal Prince Alfred Hospital, Sydney, Australia \\ e Faculty of Medicine, University of Sydney, Sydney, Australia \\ f Neuropsychology Unit, Royal Prince Alfred Hospital, Sydney, Australia
}

\section{A R T I C L E I N F O}

\section{Article history:}

Received 10 November 2009

Received in revised form 13 March 2010

Accepted 8 April 2010

Available online 23 April 2010

\section{Keywords:}

Facial expression

Orbitofrontal cortex

Approachability

Emotion

Social decision-making

Social judgements

\begin{abstract}
A B S T R A C T
Facial expressions of emotion display a wealth of important social information that we use to guide ou social judgements. The aim of the current study was to investigate whether patients with orbitofrontal cortex (OFC) lesions exhibit an impaired ability to judge the approachability of emotional faces. Furthermore, we also intended to establish whether impaired approachability judgements provided to emotional faces emerged in the presence of preserved explicit facial expression recognition. Using non-parametric statistics, we found that patients with OFC lesions had a particular difficulty using negative facial expressions to guide approachability judgements, compared to healthy controls and patients with frontal lesions sparing the OFC. Importantly, this deficit arose in the absence of an explicit facial expression recognition deficit. In our sample of healthy controls, we also demonstrated that the capacity to recognise facial expressions was not significantly correlated with approachability judgements given to emotional faces. These results demonstrate that the integrity of the OFC is critical for the appropriate assessment of approachability from negatively valenced faces and this ability is functionally dissociable from the capacity to explicitly recognise facial expressions.
\end{abstract}

(C) 2010 Elsevier Ltd. All rights reserved.
Facial expressions of emotion signal important information about the internal states of others and the external events that may have elicited the expression (Ekman, 1997). We use this information to guide our social behaviour. However, there has been limited research investigating the neural and cognitive processes that underlie an individual's capacity to use the information extracted from a facial expression to guide their social behaviour. Recently, there has been considerable research interest in the processes involved in making social decisions from an individual's facial appearance (Bar, Neta, \& Linz, 2006; Oosterhof \& Todorov, 2009; Richell et al., 2005; Todorov, Baron, \& Oosterhof, 2008; Todorov, Said, Engell, \& Oosterhof, 2008; Winston, Strange, O’Doherty, \& Dolan, 2002). These studies have typically focussed on the capacity to make social judgements, such as approachability and trustworthiness, from the faces of other individuals using faces with emotionally neutral expressions that vary naturally in their perceived approachability and trustworthiness. Healthy adults tend

\footnotetext{
* Corresponding author at: Macquarie Centre for Cognitive Science (MACCS), Macquarie University, Sydney, NSW 2109, Australia. Tel.: +61 29850 4436; fax: +6129850 6059.

E-mail address: mwillis@maccs.mq.edu.au (M.L. Willis).
}

to concur about which of these neutral faces are approachable and which are unapproachable, and those which are trustworthy and those which are not (e.g., Adolphs, Tranel, \& Damasio, 1998; Willis \& Todorov, 2006). These studies have also highlighted the potential role that the perception of emotion plays in making social judgements. For instance, studies investigating trustworthiness judgements have demonstrated that faces that are perceived to be displaying anger are considered less trustworthy than faces thought to be reflecting other emotions (Oosterhof \& Todorov, 2009; Richell et al., 2005; Winston et al., 2002). A recent study using emotionally expressive faces also revealed that negatively valenced faces (i.e., angry, sad and fearful faces) are rated as less approachable than happy faces (Porter, Coltheart, \& Langdon, 2007).

The capacity to make appropriate social judgements, in particular whether to approach or trust others, is impaired in people with evidence of abnormal amygdala functioning as a consequence of bilateral amygdala lesions, autism, schizophrenia, and Williams syndrome (Adolphs et al., 1998; Bellugi, Adolphs, Cassady, \& Chiles, 1999; Frigerio et al., 2006; Hall et al., 2004; Jones et al., 2000). Similarly, amygdala responses in healthy individuals increase as perceived trustworthiness decreases (Engell, Haxby, \& Todorov, 2007; Todorov, Said, et al., 2008; Winston et al., 2002). It has been proposed that the relationship between amygdala dysfunc- 


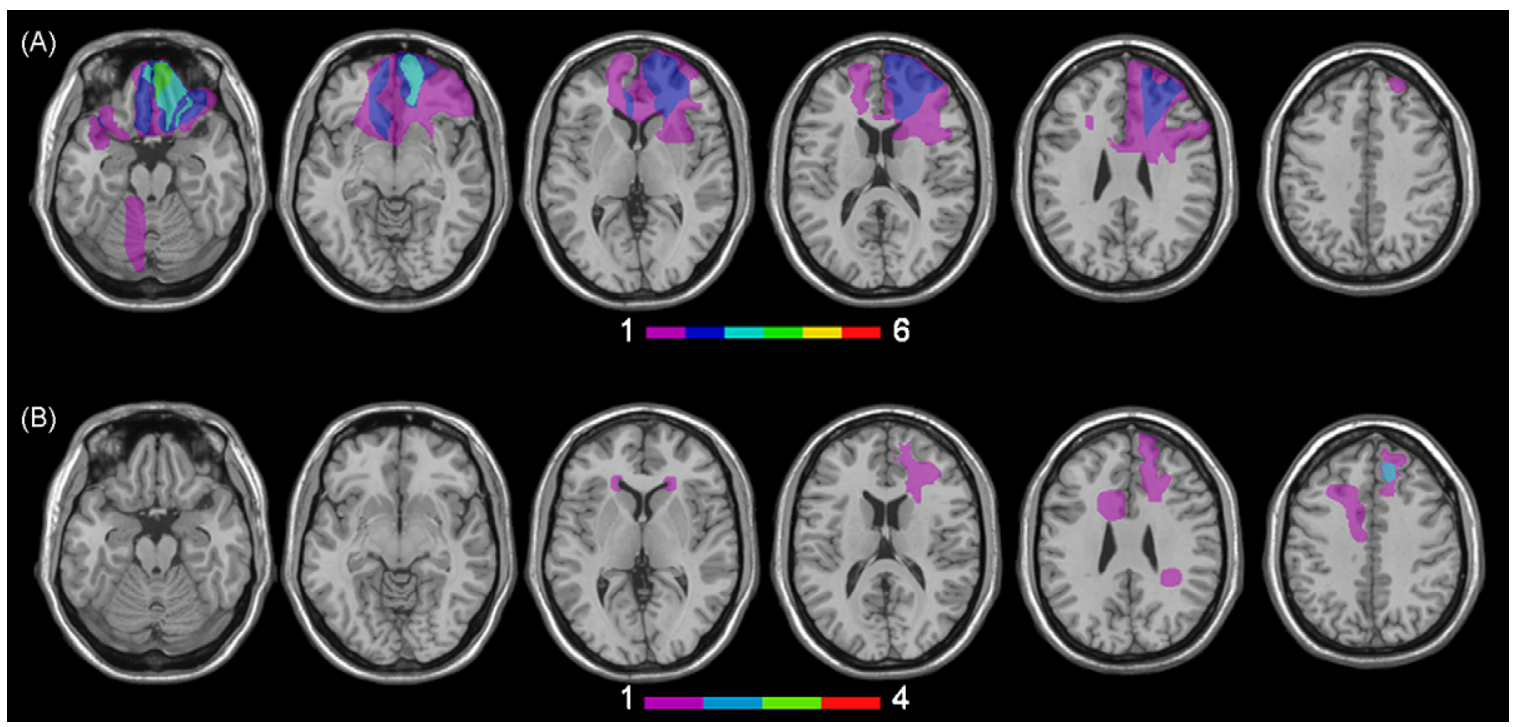

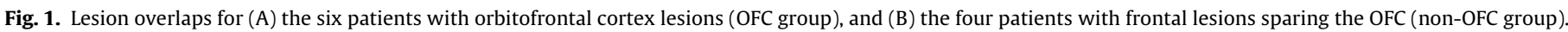
The number of subjects with lesions at each location is indicated by the colour bar at the bottom.

tion and inappropriate social judgements exists because a core component of making a social judgement involves assessing how potentially threatening the other individual is, a function that is often attributed to the amygdala (Adolphs, 2002, 2003). Moreover, it seems that differential amygdala responses to trustworthiness represent responses to differences in valence rather than trustworthiness per se (Todorov \& Engell, 2008).

The orbitofrontal cortex (OFC) occupies the ventral region of the prefrontal cortex (Kringelbach \& Rolls, 2004), which is reciprocally connected with the amygdala (Ghashghaei \& Barbas, 2002). Damage to the OFC results in impairments in social and emotional behaviour and decision-making (Beer, Heerey, Keltner, Scabini, \& Knight, 2003; Beer, Knight, \& D’Esposito, 2006; Blair \& Cipolotti, 2000; Bramham, Morris, Hornak, Bullock, \& Polkey, 2009; Damasio, Tranel, \& Damasio, 1990; Hornak et al., 2003; Hornak, Rolls, \& Wade, 1996; Rolls, Hornak, Wade, \& McGrath, 1994; Stone, BaronCohen, \& Knight, 1998). Despite the numerous anecdotal reports of impaired social and emotional functioning in individuals who have sustained damage to the OFC, no studies have systematically assessed OFC patients' capacity to use facial expressions to guide social decisions, such as judging approachability. Thus, the first aim of the current study was to establish whether OFC patients exhibit an impaired ability to use facial expressions to guide approachability judgements. We expected that they would.

The second aim was to determine whether differences in approachability judgements provided to emotional faces would be driven by impaired facial expression recognition, or whether patients with OFC lesions might be capable of recognising facial expressions explicitly but exhibit an impaired ability to use facial expressions to guide their approachability judgements appropriately. It is not clear whether the OFC is integral to the explicit recognition of facial expression; while some patient studies have shown OFC damage is associated with explicit facial expression deficits (e.g., Heberlein, Padon, Gillihan, Farah, \& Fellows, 2008; Hornak et al., 1996), others have not (Beer et al., 2003; Hornak et al., 2003). Beer et al. (2003) demonstrated inappropriate social behaviour regulation (e.g., inappropriate self-disclosure and teasing behaviour) in OFC patients, despite evidence of intact recognition of basic facial expressions. Similarly, Hornak et al. (2003) found that individuals with OFC lesions were able to recognise facial expressions, despite partners and family members reporting that the patients exhibited inappropriate responses to emotion in others in their daily lives. These findings suggest that OFC damage could result in a deficit in the utilisation of information from facial expressions to guide a social decision, like judging approachability, in the presence of unimpaired facial expression recognition.

Previous research has indicated that the OFC appears to be involved in the processing of negative facial expressions to a greater extent than that demonstrated for positive facial expressions (Iidaka et al., 2001; Ruffman, Henry, Livingstone, \& Phillips, 2008). Therefore, in the current study, we examined whether OFC patients exhibit an impaired capacity to use facial expressions to guide social decisions by assessing approachability judgements given to a set of negatively valenced facial expressions (anger, disgust, fear, and sadness) and a positive facial expression (happiness). We tested patients who had sustained OFC lesions, control patients with frontal lobe lesions sparing the OFC and healthy controls. Given previous evidence reporting socially inappropriate behaviour in individuals with OFC lesions, we predicted that the influence of facial expressions of emotion on approachability judgements would be significantly reduced in OFC patients, compared to both brain damaged and healthy controls. We anticipated that inappropriate approach behaviour would be characterised by an increased willingness to approach unfamiliar individuals displaying negative emotions. We expected all groups of participants to rate happy faces as very approachable.

After judging approachability, the participants categorised the facial expression displayed by each of the faces. This enabled us to examine whether the ability to explicitly recognise facial expressions and the capacity to use facial expressions to guide approachability judgements are dissociable processes. This was addressed in two ways.

First, by establishing whether inappropriate approachability judgements would be seen in individuals with OFC lesions in the absence of impaired facial expression recognition. As previous evidence has suggested that damage sustained to the OFC results in an impaired capacity to regulate social behaviour appropriately in response to facial expressions, despite intact facial expression recognition (e.g., Hornak et al., 2003), we hypothesised that impaired approachability judgements to emotional faces would be shown in individuals with OFC lesions in the absence of explicit facial expression recognition impairments. Second, by examining whether there was a correlation between healthy individuals' explicit facial expression recognition performance and 
Table 1

Demographic and neuropsychological profiles of OFC and non-OFC patient groups and their control groups.

\begin{tabular}{|c|c|c|c|c|c|c|c|c|c|c|}
\hline Group & & Patient & Sex & Handedness & Aetiology & Age (years) & NART Full Scale IQ & Education (years) & $\begin{array}{l}\text { Benton Test of } \\
\text { Facial } \\
\text { Recognition }\end{array}$ & $\begin{array}{l}\text { Lesion volume } \\
\left(\mathrm{cm}^{3}\right)\end{array}$ \\
\hline \multirow[t]{7}{*}{ OFC patients } & 1 & M.N. & $\mathrm{F}$ & $\mathrm{R}$ & $\mathrm{ME}$ & 58 & 102 & 15 & 48 & 342 \\
\hline & 2 & G.V. & M & $\mathrm{R}$ & $\mathrm{HI}$ & 20 & 103 & 10 & 44 & 1 \\
\hline & 3 & N.G. & $\mathrm{F}$ & $\mathrm{L}$ & $\mathrm{HI} / \mathrm{ST}^{\mathrm{a}}$ & 26 & 106 & 17 & 51 & 30 \\
\hline & 4 & P.J. & M & $\mathrm{R}$ & $\mathrm{AB}$ & 46 & 110 & 18 & 49 & 65 \\
\hline & 5 & C.O. & M & $\mathrm{R}$ & HI & 27 & 108 & 12 & 42 & 1 \\
\hline & 6 & L.L. & M & $\mathrm{R}$ & ST & 66 & 105 & 15 & 48 & 58 \\
\hline & Mean $(S D)$ & & & & & $41(19)$ & $106(3)$ & $15(3)$ & $47(3)$ & $83(130)$ \\
\hline OFC controls & Mean $(S D)$ & & & & & $41(16)$ & $108(9)$ & $15(2)$ & $48(4)$ & - \\
\hline \multirow[t]{5}{*}{ Non-OFC patients } & 1 & L.U. & M & $\mathrm{R}$ & ST & 67 & 113 & 15 & 45 & 1 \\
\hline & 2 & E.R. & M & $\mathrm{R}$ & $\mathrm{AB}$ & 49 & 103 & 13 & 46 & 21 \\
\hline & 3 & C.C. & $\mathrm{F}$ & $\mathrm{R}$ & HE & 37 & 111 & 18 & 48 & 5 \\
\hline & 4 & N.P. & $\mathrm{F}$ & $\mathrm{R}$ & ST & 57 & 108 & 18 & 51 & 2 \\
\hline & Mean $(S D)$ & & & & & $53(13)$ & $109(4)$ & $16(2)$ & $48(3)$ & $7(9)$ \\
\hline Non-OFC controls & Mean $(S D)$ & & & & & $52(13)$ & $108(10)$ & $16(3)$ & $49(4)$ & - \\
\hline
\end{tabular}

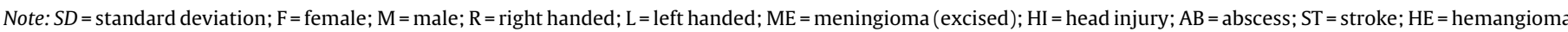
(excised); NART = National Adult Reading Test.

a This patient also had a right cerebellar tumour excised in childhood.

approachability ratings provided to emotional faces. An absence of a relationship would suggest that explicit facial expression categorisation and judgements of approachability are dissociable cognitive processes. Such a finding would highlight the need to focus more heavily on social decision-making processes when attempting to understand abnormal social behaviour.

\section{Methods}

\subsection{Participants}

The project was approved by the Ethics Review Committees at Royal Prince Alfred Hospital and Macquarie University. All participants provided their informed consent.

\subsubsection{Patient groups}

Patients were neurological outpatients recruited from Royal Prince Alfred Hospital if they had sustained a frontal lobe lesion, spoke English, were aged 20-70 and did not have receptive dysphasia. MRI or CT scans were obtained for all patients and were reviewed by a radiologist (K.M.) who was unaware of the cognitive results. K.M. mapped the lesion locations onto brain templates and determined lesion volume based on maximum anterior/posterior, ventral/dorsal and medial/lateral extent. Fig. 1 presents all the lesions overlapped for the six patients with OFC lesions (OFC group) and four without OFC lesions (non-OFC group). There was no significant difference between lesion volume for the OFC and non-OFC patient groups (Mann-Whitney $U$-test $=7, p=0.342$ ). Both groups consisted of patients with a variety of aetiologies, with the most common being stroke.

\subsubsection{Healthy controls}

Thirty-six control participants (20 female) aged between 20 and 70 years $(M=44.17, S D=15.8)$ with no history of neurological or psychiatric problems were assessed. As some studies have demonstrated sex differences in emotion recognition performance (e.g., Montagne, Kessels, Frigerio, de Haan, \& Perrett, 2005), we performed independent samples $t$-tests to investigate whether there was evidence of any sex differences in performance on the emotion recognition and approachability tasks. No significant sex differences emerged for emotion recognition performance or approachability ratings ( $t(34)<1$, for all comparisons). An absence of sex differences is not uncommon (e.g., Palermo \& Coltheart, 2004).

As facial expression recognition has also been shown to decline with age (e.g., Calder et al., 2003) we examined the correlations between age and emotion recognition performance and approachability ratings. A significant positive correlation emerged between age and the total number of errors made when recognising negative facial expressions, reflecting poorer emotion recognition with increasing age $(r=0.686, p<0.0005)$. Similarly, age was positively correlated with mean approachability ratings given to negatively valenced faces $(r=0.397, p=0.016)$, indicating that as age increases, emotionally negative faces were associated with more positive approachability ratings. No significant correlations were observed between age and recognition performance or approachability ratings for happy faces $(p>0.515$, for both correlations).

As Table 1 shows, the mean age of the non-OFC patients was greater than that of the OFC patients. Given that expression recognition performance declines markedly with increasing age and that negatively valenced faces are judged more approachable as age increases, we opted to create two control groups that were carefully matched in age to the two patient groups. The OFC control group comprised 25 (15 female) healthy control participants with ages ranging from 20 to 69 ( $M=41.44$ $S D=16.22$ ). The non-OFC control group consisted of 24 healthy control participants (13 female) with ages ranging from 26 to $70(M=51.58, S D=13.20)$.

\subsection{Stimuli}

The stimuli were photographs of the faces of 10 unfamiliar individuals (five female), each displaying an angry, disgusted, fearful, happy, sad and neutral expression, for a total of 60 faces. In addition to positive and negative expressions, we asked participants to judge the approachability of each face with a "neutral" expression so there was a baseline rating for each individual rater, which was not influenced by emotional expression. The faces were taken from the Karolinska directed emotional faces (KDEF) database (Lundqvist, Flykt, \& Öhman, 1998). The faces (256 grey levels, $72 \mathrm{ppi}$ ) were scaled to be the same size, covering a visual angle of approximately $4.3^{\circ} \times 6.5^{\circ}$

\subsection{Procedure}

\subsubsection{Approachability task}

Participants were instructed to imagine being on a crowded street on their way to meet a friend. They were asked to pretend that they were lost and in a hurry and as a consequence, needed to ask someone for directions in order to meet their friend on time. They were asked to imagine seeing each person in the crowd and to indicate the extent to which they agreed with the following statement "I would approach this person to ask for directions." The statement was displayed above each face during the task. Responses were made on a 5-point likert scale from -2 (strongly disagree) to +2 (strongly agree), which was presented on the screen underneath the face. The stimuli were shown one at a time on a white background, in a randomised order. Each trial commenced with the presentation of a fixation cross for $500 \mathrm{~ms}$. A face was then presented in the centre of the screen along with the statement and response scale. Participants selected their response from the response scale by mouse click. The stimuli, statement and response scale remained on the screen until a response was made. Following each response, an inter-trial interval occurred for $500 \mathrm{~ms}$.

\subsubsection{Emotion recognition task}

After completing the approachability task, we then assessed participants' ability to recognise facial expressions. Participants were shown each of the faces seen in the approachability task again. This time, they were asked to indicate whether the expression displayed on the face was angry, disgusted, fearful, happy, neutral or sad by selecting an emotion label from six options displayed above each face. Each face and the six emotion labels were displayed on the screen until a response was made.

Stimulus presentation was controlled using Superlab (Cedrus Corp.) and viewed on a 14 -in. monitor (screen size, $1024 \times 768$ pixels) on a Dell Laptop, at a viewing distance of approximately $60 \mathrm{~cm}$.

\subsection{Control tasks}

The National Adult Reading Test (NART, Nelson \& Willison, 1991) was used to estimate premorbid IQ. We also administered The Benton Test of Facial Recognition 
Table 2

Individual approachability ratings and recognition performance for both patient groups and group means.

\begin{tabular}{|c|c|c|c|c|c|c|c|c|c|c|}
\hline \multirow[t]{2}{*}{ Group } & & \multirow[t]{2}{*}{ Patient } & \multicolumn{2}{|c|}{$\begin{array}{l}\text { Approachability ratings } \\
\text { (difference scores) }\end{array}$} & \multicolumn{3}{|c|}{ Approachability ratings (raw scores) } & \multicolumn{3}{|c|}{ Recognition performance (errors) } \\
\hline & & & Negative & Positive & Negative & Neutral & Positive & Negative & Neutral & Positive \\
\hline \multirow[t]{7}{*}{ OFC patients } & 1 & M.N. & 0.25 & 0.60 & 0.25 & 0.00 & 0.60 & 7 & 0 & 0 \\
\hline & 2 & G.V. & -1.13 & 1.90 & -1.33 & -0.20 & 1.70 & 12 & 6 & 0 \\
\hline & 3 & N.G. & -1.45 & 0.80 & -1.35 & 0.10 & 0.90 & 9 & 1 & 0 \\
\hline & 4 & P.J. & -1.00 & 1.40 & -1.20 & -0.20 & 1.20 & 6 & 0 & 0 \\
\hline & 5 & C.O. & -1.35 & 1.00 & -0.35 & 1.00 & 2.00 & 8 & 0 & 0 \\
\hline & 6 & L.L. & -1.08 & 1.10 & -1.18 & -0.10 & 1.00 & 9 & 0 & 1 \\
\hline & Mean $(S D)$ & & $-0.96(0.62)$ & $1.13(0.46)$ & $-.86(0.66)$ & $0.10(0.46)$ & $1.23(0.52)$ & $8.5(2.1)$ & $1.2(2.4)$ & $0.2(0.4)$ \\
\hline OFC controls & Mean $(S D)$ & & $-1.92(0.43)$ & $1.10(0.46)$ & $-1.34(0.24)$ & $0.58(0.40)$ & $1.68(0.31)$ & $6.5(4.1)$ & $0.5(1.2)$ & $0.0(0.0)$ \\
\hline \multirow[t]{5}{*}{ Non-OFC patients } & 1 & L.U. & -1.90 & 1.00 & 1.50 & 0.50 & -1.40 & 14 & 1 & 1 \\
\hline & 2 & E.R. & -1.95 & 0.60 & 1.30 & 0.70 & -1.25 & 7 & 3 & 0 \\
\hline & 3 & C.C. & -1.80 & 1.10 & 1.70 & 0.60 & -1.20 & 5 & 0 & 0 \\
\hline & 4 & N.P. & -1.28 & 0.80 & 1.00 & 0.20 & -1.08 & 6 & 1 & 0 \\
\hline & Mean $(S D)$ & & $-1.73(0.31)$ & $0.88(0.22)$ & $-1.23(0.13)$ & $0.50(0.22)$ & $1.38(0.30)$ & $8.0(4.1)$ & $1.3(1.3)$ & $0.3(0.5)$ \\
\hline Non-OFC controls & Mean $(S D)$ & & $-1.79(0.43)$ & $1.12(0.48)$ & $-1.19(0.31)$ & $0.60(0.48)$ & $1.72(0.28)$ & $8.1(4.1)$ & $0.5(1.1)$ & $0.0(0.0)$ \\
\hline
\end{tabular}

(Benton, Hamsher, Varney, \& Spreen, 1983), in which participants are asked to match different views of an unfamiliar person's face, to confirm that participants were able to perceptually discriminate faces. Based on norms provided in the manual, no control participants and no patients were impaired at this task. Mann-Whitney $U$ tests confirmed there was no significant difference between the patient groups and their control group, for age, education, NART IQ and Benton Test of Facial Recognition (all $p s>0.173$ ).

\subsection{Statistical analyses}

\subsubsection{Approachability judgements}

For approachability judgements, we calculated difference scores for each emotional facial expression by subtracting each individual's mean approachability rating for neutral faces from their mean approachability rating provided to faces of each emotional category. This was performed in order to obtain a measure of the extent to which each emotional expression affects perceived approachability, relative to each an individual's neutral baseline approachability rating. As individuals vary in the precise range of the scale that they occupy when making approachability judgements, it was important to control for these individual differences when performing between group comparisons. We then calculated the average approachability difference scores provided to negative faces (i.e., angry, disgusted, fearful and sad) and approachability difference scores obtained for positive faces (i.e., happy).

In the first analysis, we compared performance between groups on approachability judgements, with negative and positive expressions separately assessed. Statistical analyses were performed using non-parametric Mann-Whitney $U$-tests (two-tailed) because the sample sizes of the patient groups were small and unequal. First, we compared the performance of the OFC and the non-OFC groups to their matched control groups for the two conditions. Second, we compared the performance of the two patient groups. In order to compare the two patient groups, we computed $z$-scores for each condition for each patient. These were calculated with reference to the mean and standard deviation of their relevant control group, thus ensuring that age differences between the two patient groups were controlled for. We then compared $z$-scores between the two patient groups using Mann-Whitney $U$-tests.

Although there was no statistically significant difference between the lesion volumes of the two patient groups, we wanted to confirm that any differences in approachability judgements between the OFC and non-OFC patient groups were not driven by the numerically larger lesion volume of the OFC patients. We assessed the effect of lesion volume by performing a median split and comparing approachability judgements between the five patients with the largest lesions and the five patients with the smallest lesions. We then performed Mann-Whitney $U$-tests comparing $z$-scores between the two groups for approachability judgements provided to negatively valenced faces and positively valenced faces.

\subsubsection{Facial expression recognition}

For emotion recognition, we calculated a facial expression recognition score for negative faces by summing the total number of errors for negative facial expressions (i.e., angry, disgusted, fearful and sad). We also calculated the total number of errors made when recognising happy faces and neutral faces. The same between group comparisons as outlined above for approachability judgements were also performed for negative, positive and neutral emotion recognition scores.

\subsubsection{Patient group comparison between tasks}

We also investigated whether either patient group showed a significant difference in performance on the approachability task compared to the emotion recognition task. $z$-score performance was compared between tasks using Wilcoxon signed ranks tests. Approachability and emotion recognition performance was compared for negative faces, separately for the OFC patient group and the non-OFC patient group.

\subsubsection{Correlations between approachability judgements and facial expression recognition}

For all the healthy control participants combined, we performed correlations between emotion recognition scores and approachability difference scores calculated for emotionally negative faces.

\section{Results}

\subsection{Approachability judgements}

As shown in Table 2, the OFC patients provided significantly more positive approachability ratings to negative faces, compared to their matched control group (Mann-Whitney $U$-test $=5.5$, $p<0.0005)$. For approachability ratings given to positive faces, OFC patients did not differ from controls (Mann-Whitney $U$ test $=72.5, p=0.903$ ). Approachability ratings provided by non-OFC patients did not differ significantly from those of their matched control group for either negatively or positively valenced faces (Mann-Whitney $U$-test $\geq 35, p>0.427$, for both comparisons).

The OFC group rated faces with negative expressions as more approachable than the non-OFC group (Mann-Whitney $U$-test $=2$, $p=0.038$, see Fig. 2). Both patient groups were equally likely to approach faces with happy expressions (Mann-Whitney $U$-test $=7$, $p=0.352$ )

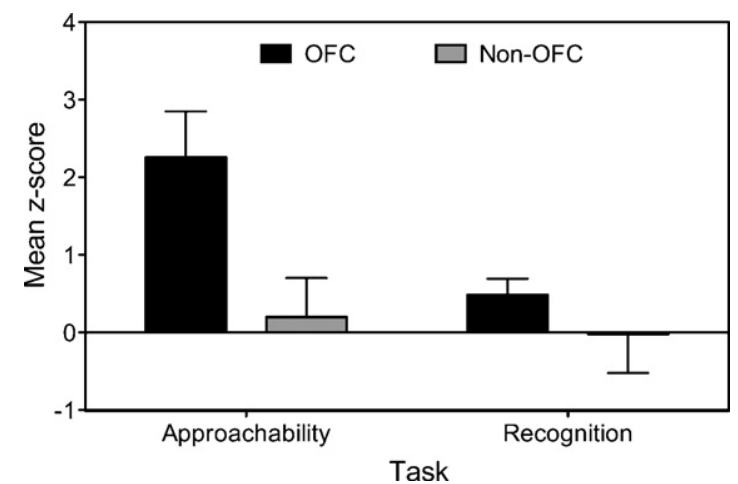

Fig. 2. Mean $z$-scores for approachability ratings and emotion recognition performance for negatively valenced faces for the OFC group and the non-OFC group. Standard error bars are shown. 
Analyses comparing the patients with the five largest lesions to those with the five smallest lesions, regardless of lesion location, indicated no significant difference between the two groups (Mann-Whitney $U$-test $\geq 7, p>0.310$, for both positive and negative expressions), thus confirming that the effects observed reflected lesion location and not lesion volume.

\subsection{Facial expression recognition}

As seen in Table 2, emotion recognition performance did not differ significantly between the OFC group and their control group for negative, positive or neutral expressions (Mann-Whitney $U$ test $\geq 46.5, p>0.158$, for all comparisons). Similarly, the non-OFC patient group's performance did not differ significantly to their control group when expression recognition performance for negative, positive and neutral expressions was considered (Mann-Whitney $U$-test $\geq 25, p>0.144$, for all comparisons). There was also no significant difference between the two patient groups for any expression (Mann-Whitney $U$-test $\geq 6, p>0.257$, for all comparisons).

\subsection{Patient group comparison between tasks}

As shown in Fig. 2, the OFC patients' mean $z$-score for negative faces was significantly larger on the approachability task than the emotion recognition task $(z=2.2, p=0.028)$, indicating that OFC patients exhibited significantly worse performance when judging the approachability of negative faces than when recognising negative emotions. In contrast, there was no significant difference between performances on the two tasks for the non-OFC patient group $(z=-0.365, p=0.715)$.

\subsection{Correlations between approachability judgements and facial expression recognition}

For all the healthy control participants combined, a significant correlation emerged between facial expression recognition performance and approachability scores for negatively valenced faces $(r=0.392, p=0.018)$. However, given that age was significantly correlated with explicit recognition and approachability judgements, it was more appropriate to perform partial correlations controlling for age, in which case no significant correlation arose between explicit recognition performance and approachability ratings for negative faces $(r=0.179, p>0.303)$. In order to establish whether there was a significant difference between the zero-order and partial correlations, we calculated Steiger's (1980) z-statistic, which confirmed that the partial correlation was significantly smaller than the zero-order correlation (Steiger's $z=-1.83, p<0.05$, onetailed). Thus, we demonstrated that when the variance explained by age is accounted for, there is a weak, non-significant relationship between explicit facial recognition performance and approachability ratings for negatively valenced emotional faces. The correlation between approachability ratings to happy faces and recognition of happy faces was not calculated, because recognition performance was at ceiling.

\section{Discussion}

In this study we investigated whether individuals with OFC damage demonstrated impaired social decision-making by assessing approachability judgements given to negative and positive faces. We also intended to establish whether there was a significant relationship between explicit facial expression recognition performance and approachability judgements provided to emotional faces.

The results revealed that OFC patients rated faces displaying negative emotional expressions as significantly more approachable than both healthy controls and patients with frontal lesions that spared the OFC. In contrast, all groups made similar approachability judgements to faces with positive expressions. This suggests that damage to the OFC affects an individual's ability to respond appropriately to negative emotion in others. This is the first study to objectively assess how facial expressions influence judgements that are pertinent to our social behaviour, in individuals with damage sustained to the OFC. While a number of previous studies have reported abnormal social behaviour following OFC damage (Beer et al., 2003, 2006; Blair \& Cipolotti, 2000; Bramham et al., 2009; Damasio et al., 1990; Hornak et al., 1996, 2003; Rolls et al., 1994; Stone et al., 1998), the current study provides strong evidence to suggest that an inability to use the facial expressions of others to guide social judgements may be an important deficit underlying the difficulties these individuals exhibit in social behaviour regulation.

A direct comparison of performance across the two tasks demonstrated that OFC patients' performance on the approachability task for negative faces was significantly worse than that displayed on the emotion recognition task for negative faces, for which OFC patients were found to exhibit no impairment relative to healthy controls. These results concur with other studies that have shown that OFC damage does not impair the ability to explicitly categorise facial expressions (Beer et al., 2003; Hornak et al., 2003). In sum, OFC damage results in an impaired ability to respond appropriately to negative facial expressions even though they can be correctly categorised. These findings converge with previous studies (Beer et al., 2003; Hornak et al., 2003) which reported evidence of impaired social behaviour regulation, despite intact facial expression recognition. Thus, the results of the current study demonstrate that OFC lesions result in a particular difficulty using negative facial expressions to guide social judgements, which does not appear to be driven by impaired explicit facial expression recognition.

We also wanted to investigate if a significant relationship existed between approachability judgements and explicit expression recognition performance in healthy controls, as the absence of a significant correlation would provide further evidence to suggest that the two abilities are functionally dissociable. An individual's capacity to recognise facial expressions of emotion was not significantly correlated with approachability judgements given to emotional faces, when age was controlled for. This result with healthy participants provides further evidence to suggest that the capacity to make social decisions from emotional faces is dissociable from facial expression recognition abilities.

Our results show that the OFC is critical for making social decisions from negative emotional faces. This may be because the OFC is particularly important for the processing of thoughts and memories of emotional events that when brought into working memory elicit a somatic state (i.e., a physiological affective state, Bechara, Damasio, \& Damasio, 2003), which guides decisionmaking (Damasio, 1994). Somatic states may play a particularly important role in the process of making approachability judgements, as such decisions cannot be guided purely by one's conceptual knowledge (as may be the case for explicit facial expression labelling tasks), but rather they require the capacity to imagine how one would feel if actually faced with the particular situation, or to recall how one felt when faced with a similar situation in the past. Such thoughts or memories presumably evoke somatic states that play an important role in guiding approachability judgements.

Individuals who have sustained damage to the OFC have been shown to lack normal physiological responses when engaged in decision-making tasks, such as the Iowa gambling task, and when processing emotional stimuli (e.g., Blair \& Cipolotti, 2000; Damasio, 1994). Therefore, we propose that the abnormal approachability judgements demonstrated by OFC patients in the current study stem from a failure to experience appropriate somatic states when 
considering how approachable negative emotional faces are. This explanation presumably accounts for the impaired social functioning that has been reported in individuals with damage to the OFC (Blair \& Cipolotti, 2000; Bramham et al., 2009; Damasio et al., 1990; Hornak et al., 1996, 2003; Rolls et al., 1994). Future studies could investigate the plausibility of this explanation by investigating physiological responses, such as skin conductance responses (SCRs), in OFC patients and controls when providing approachability judgements to emotional faces. Such a study would confirm whether SCRs emerge in healthy controls when making approachability judgements, and whether such responses would be abnormal or absent in OFC patients when making the same approachability judgements.

The findings from this study are important in developing our understanding of the deficits underlying abnormal social behaviour in individuals with OFC damage or dysfunction. The current study highlights the existence of marked deficits in the capacity to use negative facial expressions to guide approachability judgements in individuals with OFC damage, which exist in the presence of intact facial expression recognition. This finding demonstrates the need to extend beyond explicit facial expression recognition tasks in order to investigate deficits in social functioning. This is important not only for developing our understanding of the function of the OFC but also for comprehending the underlying deficits that exist in individuals within a multitude of clinical populations, such as autism, psychopathy and schizophrenia, in which abnormal social behaviour symptoms are manifest.

In sum, we have demonstrated that the OFC plays an important role in the capacity to make approachability judgements, which indicates that both the amygdala and OFC are important brain regions in a neural network that regulates social behaviour. Damage to the OFC results in an impaired ability to judge the approachability of emotionally negative faces. Furthermore, these deficits are evident in the absence of impaired facial expression recognition, which provides evidence to suggest that the capacity to use facial expressions to guide social decisions is a distinct cognitive process.

\section{Acknowledgements}

We would like to thank the patients and the control participants for participating in this project. Thanks also go to Max Coltheart and two anonymous reviewers for their comments on an earlier version of this manuscript. This research was partially supported under the Australian Research Council's Discovery Projects funding scheme (project number DP0986420).

\section{References}

Adolphs, R. (2002). Recognizing emotion from facial expressions: Psychological and neurological mechanisms. Behavioral and Cognitive Neuroscience Reviews, 1, 21-61.

Adolphs, R. (2003). Cognitive neuroscience of human social behaviour. Nature Reviews Neuroscience, 4, 165-178.

Adolphs, R., Tranel, D., \& Damasio, A. R. (1998). The human amygdala in social judgment. Nature, 393, 470-474.

Bar, M., Neta, M., \& Linz, H. (2006). Very first impressions. Emotion, 6, 269-278.

Bechara, A., Damasio, H., \& Damasio, A. R. (2003). Role of the amygdala in decisionmaking. Annals of the New York Academy of Sciences, 985, 356-369.

Beer, J. S., Heerey, E. A., Keltner, D., Scabini, D., \& Knight, R. T. (2003). The regulatory function of self-conscious emotion: Insights from patients with orbitofrontal damage. Journal of Personality and Social Psychology, 85, 594-604.

Beer, J. S., Knight, R. T., \& D'Esposito, M. (2006). Controlling the integration of emotion and cognition: The role of frontal cortex in distinguishing helpful from hurtful emotional information. Psychological Science, 17, 448-453.

Bellugi, U., Adolphs, R., Cassady, C., \& Chiles, M. (1999). Towards the neural basis for hypersociability in a genetic syndrome. NeuroReport, 10, 1653-1657.

Benton, A. L., Hamsher, K., Varney, N. R., \& Spreen, O. (1983). Contributions to neuropsychological assessment. New York: Oxford University Press.

Blair, R. J. R., \& Cipolotti, L. (2000). Impaired social response reversal: A case of 'acquired sociopathy'. Brain, 123, 1122-1141.
Bramham, J., Morris, R. G., Hornak, J., Bullock, P., \& Polkey, C. E. (2009). Social and emotional functioning following bilateral and unilateral neurosurgical prefrontal cortex lesions. Journal of Neuropsychology, 3, 125-143.

Calder, A. J., Keane, J., Manly, T., Sprengelmeyer, R., Scott, S., Nimmo-Smith, I., et al. (2003). Facial expression recognition across the adult life span. Neuropsychologia, 41, 195-202.

Damasio, A. R. (1994). Descartes' error: Emotion, reason, and the human brain. New York: Putnam.

Damasio, A. R., Tranel, D., \& Damasio, H. (1990). Individuals with sociopathic behavior caused by frontal damage fail to respond autonomically to social stimuli. Behavioural Brain Research, 41, 81-94.

Ekman, P. (1997). Should we call it expression or communication? Innovations in Social Science Research, 10, 333-344.

Engell, A. D., Haxby, J. V., \& Todorov, A. (2007). Implicit trustworthiness decisions: Automatic coding of face properties in human amygdala. Journal of Cognitive Neuroscience, 19, 1508-1519.

Frigerio, E., Burt, D. M., Gagliardi, C., Cioffi, G., Martelli, S., Perrett, D. I., et al. (2006). Is everybody always my friend? Perception of approachability in Williams syndrome. Neuropsychologia, 44, 254-259.

Ghashghaei, H. T., \& Barbas, H. (2002). Pathways for emotion: Interactions of prefrontal and anterior temporal pathways in the amygdala of the rhesus monkey. Neuroscience, $115,1261-1279$

Hall, J., Harris, J. M., Sprengelmeyer, R., Sprengelmeyer, A., Young, A. W., Santos, I. M., et al. (2004). Social cognition and face processing in schizophrenia. British Journal of Psychiatry, 185, 169-170.

Heberlein, A. S., Padon, A. A., Gillihan, S. J., Farah, M. J., \& Fellows, L. K. (2008). Ventromedial frontal lobe plays a critical role in facial emotion recognition. Journal of Cognitive Neuroscience, 20, 721-733.

Hornak, J., Rolls, E. T., \& Wade, D. (1996). Face and voice expression identification in patients with emotional and behavioural changes following ventral frontal lobe damage. Neuropsychologia, 34, 247-261.

Hornak, J., Bramham, J., Rolls, E. T., Morris, R. G., O’Doherty, J., Bullock, P. R., et al. (2003). Changes in emotion after circumscribed surgical lesions of the orbitofrontal and cingulate cortices. Brain, 126, 1691-1712.

Iidaka, T., Omori, M., Murata, T., Kosaka, H., Yonekura, Y., Okada, T., et al. (2001) Neural interaction of the amygdala with the prefrontal and temporal cortices in the processing of facial expressions as revealed by fMRI. Journal of Cognitive Neuroscience, 13, 1035-1047.

Jones, W., Bellugi, U., Lai, Z., Chiles, M., Reilly, J., Lincoln, A., et al. (2000). Hypersociability in Williams syndrome. Journal of Cognitive Neuroscience, 12(Suppl.), 30-46.

Kringelbach, M. L., \& Rolls, E. T. (2004). The functional neuroanatomy of the human orbitofrontal cortex: Evidence from neuroimaging and neuropsychology. Progress in Neurobiology, 72, 341-372.

Lundqvist, D., Flykt, A., \& Öhman, A. (1998). The Karolinska directed emotional faces-KDEF. Karolinska Institutet. ISBN:91-630r-r7164-9(CD ROM from Department of Clinical Neuroscience, Psychology section)

Montagne, B., Kessels, R. P. C., Frigerio, E., de Haan, E. H. F., \& Perrett, D. I. (2005). Sex differences in the perception of affective facial expressions: Do men really lack emotional sensitivity? Cognitive Processing, 6, 136-141.

Nelson, H., \& Willison, J. (1991). National Adult Reading Test manual (NART). Windsor, UK: NFER-Nelson.

Oosterhof, N. N., \& Todorov, A. (2009). Shared perceptual basis of emotional expressions and trustworthiness impressions from faces. Emotion, 9, 128-133.

Palermo, R., \& Coltheart, M. (2004). Photographs of facial expression: Accuracy, response times, and ratings of intensity. Behaviour Research Methods, Instruments, and Computers, 36, 634-638.

Porter, M. A., Coltheart, M., \& Langdon, R. (2007). The neuropsychological basis of hypersociability in Williams and Down syndrome. Neuropsychologia, 45, 2839-2849.

Richell, R. A., Mitchell, D. G. V., Peschardt, K. S., Winston, J. S., Leonard, A., Dolan, R. J., et al. (2005). Trust and distrust: The perception of trustworthiness of faces in psychopathic and non-psychopathic offenders. Personality and Individual Differences, 38, 1735-1744.

Rolls, E. T., Hornak, J., Wade, D., \& McGrath, J. (1994). Emotion-related learning in patients with social and emotional changes associated with frontal lobe damage. Journal of Neurology, Neurosurgery and Psychiatry, 57, 1518-1524.

Ruffman, T., Henry, J. D., Livingstone, V., \& Phillips, L. H. (2008). A meta-analytic review of emotion recognition and aging: Implications for neuropsychological models of aging. Neuroscience and Biobehavioral Reviews, 32, 863-881.

Steiger, J. H. (1980). Tests for comparing elements of a correlation matrix. Psychological Bulletin, 87, 245-251.

Stone, V. E., Baron-Cohen, S., \& Knight, R. T. (1998). Frontal lobe contributions to theory of mind. Journal of Cognitive Neuroscience, 10, 640-656.

Todorov, A., \& Engell, A. D. (2008). The role of the amygdala in implicit evaluation of emotionally neutral faces. Social Cognitive and Affective Neuroscience, 3, 303-312.

Todorov, A., Baron, S., \& Oosterhof, N. N. (2008). Evaluating face trustworthiness: A model based approach. Social, Cognitive and Affective Neuroscience, 3, 119-127.

Todorov, A., Said, C. P., Engell, A. D., \& Oosterhof, N. N. (2008). Understanding evaluation of faces on social dimensions. Trends in Cognitive Sciences, 12, 455-460.

Willis, J., \& Todorov, J. (2006). First impressions: Making up your mind after a $100-\mathrm{ms}$ exposure to a face. Psychological Science, 17, 592-598.

Winston, J. S., Strange, B. A., O'Doherty, J., \& Dolan, R. J. (2002). Automatic and intentional brain responses during evaluation of trustworthiness of faces. Nature Neuroscience, 5, 277-283. 